\title{
THE BELLMAN FUNCTIONS FOR A CERTAIN TWO-WEIGHT INEQUALITY: A CASE STUDY
}

\author{
V. VASYUNIN AND A. VOLBERG
}

\begin{abstract}
A formula is presented for the exact Bellman function of a certain "toy" two-weight problem. This adds one more function to a short list of other Bellman functions for which the precise expressions have recently been found. The case study reveals essential features of finding Bellman functions in general and gives the extremal sequences for the problem. Some open questions are posed.
\end{abstract}

\section{§0. Martingale transform: Two-weight estimate}

Let $T_{0}$ be an operator defined on some class of "sufficiently good" functions $f: \mathbb{R} \rightarrow \mathbb{R}$. Let $w, v$ be two (almost) everywhere positive locally integrable functions (from now on, we shall call such functions "weights"). The question we are going to discuss is as follows:

When is the operator $T_{0}$ bounded from $L^{2}(w)$ to $L^{2}(v)$ ?

or, in other words,

When does the inequality

$$
\int_{\mathbb{R}}\left|T_{0} f\right|^{2} v \leq C \int_{\mathbb{R}}|f|^{2} w
$$

hold true with some constant $C$ independent of $f$ ? (Unless otherwise specified, all integrals are taken with respect to standard Lebesgue measure on $\mathbb{R}$.)

Denoting $u \stackrel{\text { def }}{=} w^{-1}$, we can reformulate the above question as follows:

When is the operator $T \stackrel{\text { def }}{=} M_{\sqrt{v}} T_{0} M_{\sqrt{u}}$ bounded on $L^{2}$ ? (Here $M_{\varphi}$ stands for the operator of multiplication by $\varphi$.)

Such weighted norm inequalities arise naturally in many areas of analysis, operator theory (including the perturbation theory of selfadjoint operators), and probability theory.

The case where $w=v$ is now pretty well understood for many interesting operators $T_{0}$. For the Hilbert transform

$$
H f(t)=\frac{1}{\pi} \int_{\mathbb{R}} \frac{f(s)}{t-s} d s
$$

defined as the principal value

$$
\lim _{\varepsilon \rightarrow 0} \frac{1}{\pi} \int_{\mathbb{R} \backslash(t-\varepsilon, t+\varepsilon)} \frac{f(s)}{t-s} d s,
$$

the answer is given by the famous theorems of Helson-Szegö and Hunt-MuckenhouptWheeden.

2000 Mathematics Subject Classification. Primary 42B20, 42A50, 47B35.

Key words and phrases. Bellman function, Sawyer test, two-weight estimate.

The first author was partially supported by RFBR (grant no. 05-01-00925).

The second author was partially supported by NSF (grant DMS 0200713). 
The Helson-Szegö theorem states that the Hilbert transform is bounded on $L^{2}(v)$ if and only if the weight $v$ can be represented as $v=\exp \{\varphi+H \psi\}$, where $\varphi, \psi \in L^{\infty}$, $\|\psi\|_{L^{\infty}}<\pi / 2$.

The Hunt-Muckenhoupt-Wheeden theorem says that the Hilbert transform is bounded on $L^{2}(v)$ if and only if the weight $v$ satisfies the so-called $A_{2}$ condition,

$$
\sup _{I}\left(\frac{1}{|I|} \int_{I} v\right) \cdot\left(\frac{1}{|I|} \int_{I} v^{-1}\right)<\infty
$$

where the supremum is taken over all intervals $I \subset \mathbb{R}$.

It should be mentioned that no direct proof of the equivalence of the Helson-Szegö condition and the $A_{2}$ condition is known.

The same $A_{2}$ condition is necessary and sufficient for the weighted boundedness of the maximal function operator

$$
\mathcal{M} f(x) \stackrel{\text { def }}{=} \sup _{I \ni x} \frac{1}{|I|} \int_{I}|f|
$$

if the weights are equal, $w=v$ (here the supremum is taken over all intervals $I$ containing $x)$.

Things look much more complicated in the two-weight case. Though there exists a beautiful two-weight analog of the Helson-Szegö theorem due to Cotlar and Sadosky (see [CS]), the consensus now is that, possibly, no explicit (Muckenhoupt type) necessary and sufficient condition exists for the boundedness of the Hilbert transform.

It was a big surprise when Eric Sawyer [S1] found a necessary and sufficient condition for the boundedness of the maximal function operator $\mathcal{M}$ from $L^{2}(w)$ to $L^{2}(v)$. His theorem states that it suffices to test the boundedness on a very special class of "simple" test functions, namely, on the functions $w^{-1} \chi_{I}$ (here and below $\chi_{I}$ stands for the characteristic function of an interval $I \subset \mathbb{R}$ ). Later (see $[S 2]$ ), Sawyer presented a necessary and sufficient condition of similar type for the boundedness from $L^{2}(w)$ to $L^{2}(v)$ of certain integral operators $T_{0}$ with positive kernels. The main difference between this case and the case of the maximal function operator is that now one should also check the boundedness for the adjoint operator $T_{0}^{*}: L^{2}\left(v^{-1}\right) \rightarrow L^{2}\left(w^{-1}\right)$ on an appropriate class of simple test functions, namely, on the functions $v \chi_{I}$. The reader should not be scared by the inverses of $w$ and $v$. In most interesting cases it may be assumed that they are summable.

We can reformulate Sawyer's result in a way that gives some motivation for what follows. The criterion below applies to quite a few operators $T_{0}$ with positive kernel.

Sawyer type conditions. The operator $T=M_{\sqrt{v}} T_{0} M_{\sqrt{u}}$ is bounded on $L^{2}$ if and only if it is bounded on the test functions $\sqrt{u} \chi_{I}$ and the adjoint operator $T^{*}=M_{\sqrt{u}} T_{0}^{*} M_{\sqrt{v}}$ is bounded on the test functions $\sqrt{v} \chi_{I}$.

Throughout, conditions of this kind will be called the Sawyer type conditions. In the paper NTV1, the main aim was to present a uniform approach to recovering many of Sawyer's results (at least in $L^{2}$ ) and to obtaining at least one new theorem of Sawyer type: a necessary and sufficient condition for the boundedness of Haar multipliers. As far as we know, it was the first theorem of Sawyer type for singular integral operators: all earlier results concerned operators with positive kernels only. The Bellman function method was used in [NTV1] actually, the 1995 preprint version of that paper was the place where the harmonic analysis Bellman function appeared for the first time - except, of course, the famous Burkholder paper $\mathrm{B}$, which was implicitly related to the Bellman function technique, but employed another language.

In what follows, we shall write $\langle f\rangle_{I}$ for the average $\frac{1}{|I|} \int_{I} f$ of a function $f: \mathbb{R} \rightarrow \mathbb{R}$ over an interval $I \subset \mathbb{R}$. 
Let $\mathcal{D}$ be some dyadic lattice, and let $h_{J}(J \in \mathcal{D})$ be the corresponding family of Haar functions normalized in $L^{2}$, namely, $h_{J}=\left(\chi_{J^{+}}-\chi_{J^{-}}\right) / \sqrt{|J|}$, where the $J_{ \pm}$denote the right and the left half of $J$. We put $H_{J} \stackrel{\text { def }}{=} \sqrt{|J|} h_{J}$, and $\Delta_{J} f \stackrel{\text { def }}{=}\langle f\rangle_{J^{+}}-\langle f\rangle_{J^{-}}$.

Let $\mathbf{a}$ denote a bounded sequence $a_{J}$ enumerated by dyadic intervals. We define an operator $T_{0}(\mathbf{a})$ by

$$
T_{0}(\mathbf{a}) f \stackrel{\text { def }}{=} \sum_{J \in \mathcal{D}} a_{J}\left(f, h_{J}\right)_{L^{2}} h_{J}=\sum_{J \in \mathcal{D}} a_{J}\left[\Delta_{J} f\right] H_{J} .
$$

A Sawyer type question is whether the "weighted operator"

$$
T(\mathbf{a}) \stackrel{\text { def }}{=} M_{\sqrt{v}} T_{0}(\mathbf{a}) M_{\sqrt{u}}
$$

is bounded on $L^{2}$ if and only if it is bounded on the test functions $\sqrt{u} \chi_{I}$ and the adjoint operator $T(\mathbf{a})^{*}=M_{\sqrt{u}} T_{0}(\mathbf{a}) M_{\sqrt{v}}$ is bounded on the test functions $\sqrt{v} \chi_{I}(I \in \mathcal{D})$.

The answer is "Yes", and this was proved in [NTV1] for the family $T(\mathbf{a})$ as a whole (this means that the Sawyer type conditions are fulfilled uniformly in a if and only if all operators $T(\mathbf{a})$ are bounded uniformly). In the following statement $\boldsymbol{\sigma}$ means an arbitrary sequence of signs.

Theorem (Sawyer type theorem for Haar multipliers). The operators $T(\boldsymbol{\sigma a})$ are uniformly bounded in $L^{2}$, i.e., $\sup _{\boldsymbol{\sigma}}\|T(\boldsymbol{\sigma} \mathbf{a})\|_{L^{2} \rightarrow L^{2}}<+\infty$, if and only if they are uniformly bounded on the test functions $\sqrt{u} \chi_{I}$ and the adjoint operators $T(\boldsymbol{\sigma} \mathbf{a})^{*}$ are uniformly bounded on the test functions $\sqrt{v} \chi_{I}$, that is, if and only if there exists a constant $C>0$ such that

$$
\int_{\mathbb{R}}\left[T_{0}(\boldsymbol{\sigma} \mathbf{a})\left(w \chi_{I}\right)\right]^{2} v \leq C \int_{I} w \quad \text { and } \quad \int_{\mathbb{R}}\left[T_{0}(\boldsymbol{\sigma} \mathbf{a})\left(v \chi_{I}\right)\right]^{2} w \leq C \int_{I} v
$$

for all dyadic intervals $I \in \mathcal{D}$ and sign sequences $\boldsymbol{\sigma}$.

One can ask, of course, whether this result is true for an individual $T_{0}(\mathbf{a})$. The answer is positive; see NTV2.

Another natural question arises: whether a simpler condition can be found? Here are some necessary conditions:

(1) $\langle u\rangle_{I}\langle v\rangle_{I} \leq \frac{1}{2} \mathrm{Ca}_{I}^{-2}$ (Muckenhoupt type condition);

$\left(2_{u}\right) \frac{1}{|I|} \sum_{J \subset I} a_{J}^{2}\left[\Delta_{J} u\right]^{2}\langle v\rangle_{J}|J| \leq C\langle u\rangle_{I}$;

$\left(2_{v}\right) \frac{1}{|I|} \sum_{J \subset I} a_{J}^{2}\left[\Delta_{J} v\right]^{2}\langle u\rangle_{J}|J| \leq C\langle v\rangle_{I}$.

Indeed, fixing some $I \in \mathcal{D}$ and taking the average of $\int_{\mathbb{R}}\left[T_{0}(\boldsymbol{\sigma a})\left(u \chi_{I}\right)\right]^{2} v$ over all possible sign sequences $\sigma$, we get

$$
\frac{1}{|I|} \sum_{J \in \mathcal{D}} a_{J}^{2}\left[\Delta_{J}\left(u \chi_{I}\right)\right]^{2}\langle v\rangle_{J}|J|=\operatorname{Avrg}_{\boldsymbol{\sigma}} \frac{1}{|I|} \int_{\mathbb{R}}\left[T_{0}(\boldsymbol{\sigma} \mathbf{a})\left(u \chi_{I}\right)\right]^{2} v \leq C\langle u\rangle_{I} .
$$

Observing that $\Delta_{J}\left(u \chi_{I}\right)=\Delta_{J} u$ for all $J \subset I$ and that every square is nonnegative, we immediately arrive at $\left(2_{u}\right)$.

To prove (1), we apply the above inequality to $u \chi_{I^{ \pm}}$in place of $u$ and take only one summand corresponding to $J=I$. Since $\left|\Delta_{I}\left(u \chi_{I^{ \pm}}\right)\right|=\langle u\rangle_{I^{ \pm}}$and $\left\langle u \chi_{I^{ \pm}}\right\rangle_{I}=\frac{1}{2}\langle u\rangle_{I^{ \pm}}$, we find

i.e.,

$$
a_{I}^{2}\langle u\rangle_{I^{ \pm}}^{2}\langle v\rangle_{I} \leq \frac{1}{2} C\langle u\rangle_{I^{ \pm}}
$$

$$
\langle u\rangle_{I^{ \pm}}\langle v\rangle_{I} \leq \frac{1}{2} C a_{I}^{-2} .
$$


Recalling that $\langle u\rangle_{I} \leq \max \left\{\langle u\rangle_{I^{-}},\langle u\rangle_{I^{+}}\right\}$, we get (1). The proof of $\left(2_{v}\right)$ is similar to that of $\left(2_{u}\right)$.

Condition (1) becomes the famous Hunt-Muckenhoupt-Wheeden condition when $v=$ $w=u^{-1}$ (we have used the relationship between $u$ and $w$ introduced above). So, it is natural to ask whether this assumption (1) alone is sufficient for the two-weight boundedness. This is not the case; see $[\mathrm{N}]$. Here it is worthwhile to mention that the proof in $[\mathrm{N}]$ is also a Bellman function proof. We also note that $(1)$ and $\left(2_{u}\right)$ together are not sufficient, as another Bellman function (actually, its tending to infinity) shows; see [NV].

However, we are going to show that (1) alone is sufficient for the "bilinear test of Sawyer".

\section{§1. Bilinear teSt}

Now, let a denote a bounded sequence $a_{J}$ enumerated by dyadic intervals and such that each $a_{J}$ is \pm 1 . As has already been said, we are reduced to considering Sawyer tests with $C$ independent of $J$ :

$$
\begin{aligned}
& \sup _{a}\left\|M_{\sqrt{v}} T_{0}(\mathbf{a}) M_{\sqrt{u}} \sqrt{u} \chi_{J}\right\|_{2} \leq C\left\|\sqrt{u} \chi_{J}\right\|_{2}=C\langle u\rangle_{J}^{1 / 2}|J|^{1 / 2}, \\
& \sup _{a}\left\|M_{\sqrt{u}} T_{0}(\mathbf{a}) M_{\sqrt{v}} \sqrt{v} \chi_{J}\right\|_{2} \leq C\left\|\sqrt{v} \chi_{J}\right\|_{2}=C\langle v\rangle_{J}^{1 / 2}|J|^{1 / 2} .
\end{aligned}
$$

We have already mentioned that the simple condition $\langle u\rangle_{I}\langle v\rangle_{I} \leq Q<\infty$ for all dyadic I does not ensure these estimates.

However, we consider a simpler test, where our operator is applied simultaneously to both of these test functions $\sqrt{u} \chi_{J}, \sqrt{v} \chi_{J}$. In other words, now we want that the following condition be fulfilled:

$$
\sup _{\mathbf{a}}\left|\left(M_{\sqrt{v}} T_{0}(\mathbf{a}) M_{\sqrt{u}} \sqrt{u} \chi_{J}, \sqrt{v} \chi_{J}\right)_{L^{2}}\right| \leq C\langle u\rangle_{J}^{1 / 2}\langle v\rangle_{J}^{1 / 2}|J| .
$$

Theorem 1. The assumption $\sup _{I \in \mathcal{D}}\langle u\rangle_{I}\langle v\rangle_{I} \leq M^{2}$ implies (1.1).

A closer look at the sum on the left-hand side of (1.1) shows that the most essential contribution is given by the sum over all the dyadic subintervals of $J$. Namely, we are forced to consider the inequality

$$
\frac{1}{|J|} \sum_{I \in \mathcal{D}, I \subset J}\left|\langle u\rangle_{I^{+}}-\langle u\rangle_{I^{-}}\right|\left|\langle v\rangle_{I^{+}}-\langle v\rangle_{I^{-}}\right||I| \leq C\langle u\rangle_{J}^{1 / 2}\langle v\rangle_{J}^{1 / 2} .
$$

Obviously, the next theorem proves Theorem 1.

Theorem 2. The assumption $\sup _{I \in \mathcal{D}}\langle u\rangle_{I}\langle v\rangle_{I} \leq M^{2}$ implies (1.2) with $C=16 M$.

Actually, our main goal is to investigate inequality (1.2) completely, to find its Bellman function precisely, and to find the "extremal" $u, v$ (there will be no extremal pairs, but we shall find extremal sequences of pairs). We believe that this goal is somewhat instructive.

\section{§2. Notation AND DEFInitions}

From the outset, we would like to emphasize that we shall consider arbitrary partitions of an interval rather than only dyadic ones. Moreover, we shall compare the "dyadic" and "nondyadic" cases, because we know that sometimes this technique gives drastically different results for these cases, but in the problem in question it turns out that the estimates in both cases are the same. 
We shall say that an interval $I$ and a pair of positive numbers $\alpha^{ \pm}\left(\alpha^{+}+\alpha^{-}=1\right)$ generate a pair of subintervals $I^{+}$and $I^{-}$if $\left|I^{ \pm}\right|=\alpha^{ \pm}|I|$ and $I=I^{-} \cup I^{+}$. With every interval $J$ and every sequence

$$
\left\{\alpha_{n, m}: 0 \leq m<2^{n}, 0<n<\infty\right\}
$$

such that $0<\alpha_{n, m}<1$ and $\alpha_{n, 2 k}+\alpha_{n, 2 k+1}=1$, we associate a family $\mathcal{I}$ of intervals $\left\{I_{n, m}\right\}$ such that any interval $I_{n, m}$ generates the pair $I_{n+1,2 m}=I_{n, m}^{-}, I_{n+1,2 m+1}=I_{n, m}^{+}$ with $\alpha^{-}=\alpha_{n+1,2 m}, \alpha^{+}=\alpha_{n+1,2 m+1}$, starting with $I_{0,0}=J$. The symbol $\mathcal{I}=\mathcal{I}(\alpha)$ will denote the families of subintervals of $J$ corresponding to a fixed choice of the numbers $\alpha_{n, m}$. For the special choice $\alpha_{n, m}=\frac{1}{2}$, we get the dyadic family $\mathcal{I}=\mathcal{D}$. Every family $\mathcal{I}$ has its own set of Haar functions:

$$
h_{I}(t)=\left\{\begin{array}{cl}
-\sqrt{\frac{\alpha^{+}}{\alpha^{-}|I|}} & \text { if } t \in I^{-}, \\
\sqrt{\frac{\alpha^{-}}{\alpha^{+}|I|}} & \text { if } t \in I^{+},
\end{array}\right.
$$

for $I \in \mathcal{I}$. If the family $\mathcal{I}$ is such that the maximal length of the $n$th generation intervals (i.e., $\max \left\{\left|I_{n, m}\right|: 0 \leq m<2^{n}\right\}$ ) tends to 0 as $n \rightarrow \infty$, then the Haar family forms an orthonormal basis in the space $L^{2}(J) \ominus\{$ const $\}$.

Definition. We define a domain in $\mathbb{R}^{2}$ :

$$
\Omega=\Omega_{m, M}=\left\{x=\left(x_{1}, x_{2}\right): x_{i} \geq 0, m^{2} \leq x_{1} x_{2} \leq M^{2}\right\},
$$

and a function on this domain:

$$
\mathbf{B}(x)=\mathbf{B}(x ; m, M)=\sup _{\mathcal{I}, u, v}\left\{\frac{1}{|J|} \sum_{I \in \mathcal{I}}\left|\left(u, h_{I}\right)\right|\left|\left(v, h_{I}\right)\right|\right\},
$$

where the supremum is taken over all possible partitions $\mathcal{I}$ of the interval $J$, and over all nonnegative functions $u, v$ in $L^{1}(J)$ such that $\left(\langle u\rangle_{J},\langle v\rangle_{J}\right)=\left(x_{1}, x_{2}\right)=x$ and the inequalities

$$
m^{2} \leq\langle u\rangle_{I}\langle v\rangle_{I} \leq M^{2}
$$

are fulfilled for all subintervals $I$ of $J$.

The function $\mathbf{B}$ is called a Bellman function.

If we fix a partition $\mathcal{I}=\mathcal{D}$ and consider the supremum of the same sum over all pairs $u, v$ with the restriction (2.1) only for the dyadic intervals $I$, we get the dyadic Bellman function $\mathbf{B}_{\mathcal{D}}$.

Remark 1. Recall that

$$
\begin{aligned}
\left|\left(u, h_{I}\right)\right|\left|\left(v, h_{I}\right)\right| & =\alpha^{+} \alpha^{-}|I|\left|\langle u\rangle_{I^{+}}-\langle u\rangle_{I^{-}}\right|\left|\langle v\rangle_{I^{+}}-\langle v\rangle_{I^{-}}\right| \\
& =\alpha^{+} \alpha^{-}|I|\left|\Delta_{I} u\right|\left|\Delta_{I} v\right| .
\end{aligned}
$$

Remark 2. The function $\mathbf{B}$ does not depend on the initial interval $J$. Indeed, consider two different intervals $J$ and $J^{\prime}$. The linear mapping of $I$ onto $I^{\prime}$ maps $L^{1}(J)$ onto $L^{1}\left(J^{\prime}\right)$ and the family of intervals $\mathcal{I}$ onto the family $\mathcal{I}^{\prime}$, not changing the averages $\langle u\rangle_{I}=\left\langle u^{\prime}\right\rangle_{I^{\prime}}$ and the splitting coefficients $\alpha_{I}^{ \pm}=\alpha_{I^{\prime}}^{ \pm}$. Recalling the relation from the preceding remark, we see that the supremum in the definition of the function $\mathbf{B}$ is taken over the same set of numbers not depending on the choice of $J$.

Remark 3. Since for $m=M$ only constant functions $u$ and $v$ can satisfy (2.1), we have $\mathbf{B}(x ; m, m)=\mathbf{B}_{\mathcal{D}}(x ; m, m)=0$. Thus, in all our calculations we may assume that $m<M$. 
Remark 4. For an arbitrary point $x \in \Omega$, there exists a pair of functions $u, v$ satisfying (2.1) and such that $\left(\langle u\rangle_{J},\langle v\rangle_{J}\right)=\left(x_{1}, x_{2}\right)=x$, i.e., the supremum in the definition of $\mathbf{B}$ is taken over a nonempty set. Choosing the partition $J=J^{+} \cup J^{-}$with the splitting coefficients

$$
\alpha^{-}=\frac{M-\sqrt{x_{1} x_{2}}}{M-m}, \quad \alpha^{+}=\frac{\sqrt{x_{1} x_{2}}-m}{M-m},
$$

we take a pair of step functions constant on $J^{ \pm}$:

$$
u(t)=\left\{\begin{array}{ll}
M \sqrt{\frac{x_{1}}{x_{2}}} & t \in J^{+} \\
m \sqrt{\frac{x_{1}}{x_{2}}} & t \in J^{-}
\end{array} \quad \text { and } \quad v(t)=\left\{\begin{array}{ll}
M \sqrt{\frac{x_{2}}{x_{1}}} & t \in J^{+} \\
m \sqrt{\frac{x_{2}}{x_{1}}} & t \in J^{-}
\end{array} .\right.\right.
$$

It is easy to check that $\left(\langle u\rangle_{J},\langle v\rangle_{J}\right)=x$. The geometrical picture makes the situation absolutely transparent: our initial point $x$ belongs to the segment of the straight line with the endpoints $\left(M \sqrt{x_{1} / x_{2}}, M \sqrt{x_{2} / x_{1}}\right),\left(m \sqrt{x_{1} / x_{2}}, m \sqrt{x_{2} / x_{1}}\right)$, which are on the boundary of the strip $\Omega$ situated between two hyperbolas. This segment lies on the ray starting at the origin; therefore, the entire segment is inside of $\Omega$. Since, clearly, the averages $\left(\langle u\rangle_{I},\langle v\rangle_{I}\right)$ over any subinterval $I \subset J$ are on the same segment, condition (2.1) is fulfilled for all $I \subset J$.

\section{§3. MAIN RESULT}

Now we are ready to state the main result of the paper.

\section{Theorem 3.}

$$
\mathbf{B}(x ; m, M)=\mathbf{B}_{\mathcal{D}}(x ; m, M)=4 M \sqrt{x_{1} x_{2}}-x_{1} x_{2}+m^{2}-4 m M .
$$

Remark 5. To make the statement simpler, we did not include an important detail in it; namely, identity (3.1) will be proved for all points $x \in \Omega$ in the dyadic case, i.e., for the function $\mathbf{B}_{\mathcal{D}}$, but for the function $\mathbf{B}$ it will be proved for all points except the points on the upper boundary, i.e., for $x=\left(x_{1}, x_{2}\right)$ with $m^{2} \leq x_{1} x_{2}<M^{2}$. For the points on the upper boundary we are only able to prove the inequality

$$
\mathbf{B}(x ; m, M) \leq 3 M^{2}-m^{2}-4 m M .
$$

We cannot assert that equality occurs here, and that the function is continuous on the upper boundary of its domain. We shall discuss this problem later, after the proof.

We get Theorem 2 immediately if we put $m=0$ in Theorem 3 and take into account the relation mentioned in Remark 1 with $\alpha^{ \pm}=\frac{1}{2}$. Moreover, if the pair $u, v$ satisfies condition (2.1) not only for dyadic but for arbitrary subintervals of the initial interval $J$, then the same inequality is true for the sum taken not necessarily over a dyadic but over an arbitrary partition $J$, i.e., the following is true.

Corollary 1. The assumption $\sup _{I \subset J}\langle u\rangle_{I}\langle v\rangle_{I} \leq M^{2}$ implies

$$
\frac{1}{|J|} \sum_{I \in \mathcal{I}}\left|\left(u, h_{I}\right)\right|\left|\left(v, h_{I}\right)\right| \leq 4 M\langle u\rangle_{J}^{1 / 2}\langle v\rangle_{J}^{1 / 2}
$$

for an arbitrary partition $\mathcal{I}$.

\section{§4. How to Find the Bellman function}

This section can be skipped by the reader who is interested in the result itself. The considerations presented here will not be used in the formal proof of the theorem; however, without them the origin of identity (3.1) would be unclear.

First, we state a certain property of the Bellman function that will help us to find it. 
Lemma 1. There exists a function $\varphi$ of one variable with the following properties: it is defined on the interval $\left[m^{2}, M^{2}\right]$ and satisfies the boundary condition $\varphi\left(m^{2} ; m, M\right)=0$, and we have the formula $\mathbf{B}(x ; m, M)=\varphi\left(x_{1} x_{2} ; m, M\right)$.

Proof. For any admissible pair of functions $u, v$ (i.e., for a pair satisfying condition (2.1)) and an arbitrary positive number $t$, we introduce another pair $\tilde{u}=t u$ and $\tilde{v}=t^{-1} v$. Since $\langle u\rangle_{I}\langle v\rangle_{I}=\langle\tilde{u}\rangle_{I}\langle\tilde{v}\rangle_{I}$, the pairs $u, v$ and $\tilde{u}, \tilde{v}$ run over the set of all admissible pairs simultaneously. Summing the identities $\left(u, h_{I}\right)\left(v, h_{I}\right)=\left(\tilde{u}, h_{I}\right)\left(\tilde{v}, h_{I}\right)$ and taking the supremum over the set of all admissible pairs, we arrive at the relation

$$
\mathbf{B}(x)=\mathbf{B}(\tilde{x})=\mathbf{B}\left(t x_{1}, \frac{1}{t} x_{2}\right) .
$$

Choosing $t=x_{2}$, we get

$$
\mathbf{B}\left(x_{1}, x_{2}\right)=\mathbf{B}\left(x_{1} x_{2}, 1\right) \stackrel{\text { def }}{=} \varphi\left(x_{1} x_{2}\right),
$$

where

$$
\varphi(s ; m, M) \stackrel{\text { def }}{=} \mathbf{B}(s, 1 ; m, M) .
$$

Furthermore, for an admissible pair $u, v$ the relation $x_{1} x_{2}=m^{2}$ occurs if and only if $u=$ const, $v=$ const, and $u v=m^{2}$. Indeed, this is a consequence of the convexity of the domain $\left\{\left(x_{1}, x_{2}\right): x_{i}>0, x_{1} x_{2} \geq m^{2}\right\}$ : the inequalities $x_{1}^{ \pm} x_{2}^{ \pm} \geq m^{2}$ together with the identities $x_{i}=\alpha^{+} x_{i}^{+}+\alpha^{-} x_{i}^{-}, x_{1} x_{2}=m^{2}$ imply that all three points coincide: $x=x^{+}=x^{-}$. This means that the average of $u$ and $v$ is the same for all intervals $I \subset J$, so that they are constant functions and

$$
\varphi\left(m^{2} ; m, M\right)=\mathbf{B}(u v, 1 ; m, M)=\mathbf{B}(u, v ; m, M)=0,
$$

since for constant functions $u, v$ we have $\Delta_{I} u=\Delta_{I} v=0$ for all intervals $I$.

Now we deduce a basic inequality for the dyadic Bellman function $\mathbf{B}_{\mathcal{D}}$.

Lemma 2. Let $x^{ \pm}$be two arbitrary points in the ("hyperbolic") strip $\Omega$ such that their arithmetic mean, i.e., the point $x=\frac{1}{2}\left(x^{+}+x^{-}\right)$, also belongs to $\Omega$. Then

$$
\mathbf{B}_{\mathcal{D}}(x) \geq \frac{1}{2}\left(\mathbf{B}_{\mathcal{D}}\left(x^{+}\right)+\mathbf{B}_{D}\left(x^{-}\right)\right)+\frac{1}{4}\left|x_{1}^{+}-x_{1}^{-}\right|\left|x_{2}^{+}-x_{2}^{-}\right| .
$$

Proof. We take two pairs of functions $u^{ \pm}, v^{ \pm}$defined on $J^{ \pm}$(respectively) and satisfying (2.1) for all dyadic subintervals of $J^{ \pm}$and "glue" them to obtain a pair $u, v$ defined on $J$ :

$$
u(t)=\left\{\begin{array}{ll}
u^{+}(t) & \text { if } t \in J^{+} \\
u^{-}(t) & \text { if } t \in J^{-}
\end{array} \text {and } \quad v(t)= \begin{cases}v^{+}(t) & \text { if } t \in J^{+} \\
v^{-}(t) & \text { if } t \in J^{-}\end{cases}\right.
$$

Now we split the sum in the definition of the function $\mathbf{B}_{\mathcal{D}}$ into three parts:

$$
\begin{aligned}
\frac{1}{|J|} \sum_{I \in \mathcal{D}}\left|\left(u, h_{I}\right)\right|\left|\left(v, h_{I}\right)\right| \\
=\frac{1}{2\left|J^{+}\right|} \sum_{I \in \mathcal{D}^{+}}\left|\left(u, h_{I}\right)\right|\left|\left(v, h_{I}\right)\right| \\
\quad+\frac{1}{2\left|J^{-}\right|} \sum_{I \in \mathcal{D}^{-}}\left|\left(u, h_{I}\right)\right|\left|\left(v, h_{I}\right)\right|+\frac{1}{|J|}\left|\left(u, h_{J}\right)\right|\left|\left(v, h_{J}\right)\right|,
\end{aligned}
$$

where the $\mathcal{D}^{ \pm}$are dyadic subintervals of $J^{ \pm}$. Note that $\frac{1}{|J|}\left|\left(u, h_{J}\right)\right|\left|\left(v, h_{J}\right)\right|=\frac{1}{4}\left|x_{1}^{+}-x_{1}^{-}\right| \times$ $\left|x_{2}^{+}-x_{2}^{-}\right|$and the pair $u, v$ runs over a subset of the set of admissible pairs for $\mathbf{B}_{\mathcal{D}}(x)$ as $u^{ \pm}, v^{ \pm}$run over the set of all admissible pairs for $\mathbf{B}_{\mathcal{D}}\left(x^{ \pm}\right)$. Passing to the supremum over this set in (4.2), we arrive at the desired inequality. 
Remark 6 . We emphasize that, in the nondyadic case, the pair $u, v$ constructed above may fail to be admissible, i.e., may fail to satisfy (2.1) for every $I \subset J$ in spite of the fact that (2.1) is true for all $I \subset J^{+}$or $I \subset J^{-}$. Nevertheless, viewing this as a heuristic assumption, we can look for a function $B$ satisfying a similar inequality for arbitrary splitting coefficients:

$$
B(x) \geq \alpha^{+} B\left(x^{+}\right)+\alpha^{-} B\left(x^{-}\right)+\alpha^{+} \alpha^{-}\left|x_{1}^{+}-x_{1}^{-}\right|\left|x_{2}^{+}-x_{2}^{-}\right|,
$$

where $x=\alpha^{+} x^{+}+\alpha^{-} x^{-}$; after such a function $B$ is found, we shall be able to prove that $B=\mathbf{B}$.

Remark 7. Inequalities (4.1) and (4.3) have the following infinitesimal form:

$$
-\left(\frac{\partial^{2} B}{\partial x_{1}^{2}} \Delta_{1}^{2}+2 \frac{\partial^{2} B}{\partial x_{1} \partial x_{2}} \Delta_{1} \Delta_{2}+\frac{\partial^{2} B}{\partial x_{2}^{2}} \Delta_{2}^{2}\right) \geq 2\left|\Delta_{1} \Delta_{2}\right|,
$$

where, with a slight abuse of notation, we write $\Delta_{i}$ for $x_{i}^{+}-x_{i}^{-}$. In fact, we always assume that $x_{1}$ is the average of a certain summable function $u$ over some interval $I$, i.e., $\Delta_{1}=\Delta_{I} u$, and similarly $\Delta_{2}=\Delta_{I} v$.

To deduce (4.4) from (4.3), we use the relations

$$
x_{i}^{+}=x_{i}+\alpha^{-} \Delta_{i} \text { and } x_{i}^{-}=x_{i}-\alpha^{+} \Delta_{i}
$$

and, assuming that the function $B$ is sufficiently smooth, take three terms of the Taylor expansion:

$$
B\left(x^{ \pm}\right) \approx B(x) \pm \alpha^{\mp} \sum_{i=1}^{2} \frac{\partial B}{\partial x_{i}} \Delta_{i}+\frac{1}{2}\left(\alpha^{\mp}\right)^{2} \sum_{i, j=1}^{2} \frac{\partial^{2} B}{\partial x_{i} \partial x_{j}} \Delta_{i} \Delta_{j}
$$

whence

$$
\alpha^{+} B\left(x^{+}\right)+\alpha^{-} B\left(x^{-}\right) \approx B(x)+\frac{1}{2} \alpha^{+} \alpha^{-} \sum_{i, j=1}^{2} \frac{\partial^{2} B}{\partial x_{i} \partial x_{j}} \Delta_{i} \Delta_{j},
$$

which yields (4.4) immediately.

Remark 8. Condition (4.4) contains two matrix inequalities:

$$
\begin{aligned}
& \left(\begin{array}{cc}
\frac{\partial^{2} B}{\partial x_{1}^{2}} & \frac{\partial^{2} B}{\partial x_{1} \partial x_{2}}-1 \\
\frac{\partial^{2} B}{\partial x_{1} \partial x_{2}}-1 & \frac{\partial^{2} B}{\partial x_{2}^{2}}
\end{array}\right) \leq 0 \\
& \left(\begin{array}{cc}
\frac{\partial^{2} B}{\partial x_{1}^{2}} & \frac{\partial^{2} B}{\partial x_{1} \partial x_{2}}+1 \\
\frac{\partial^{2} B}{\partial x_{1} \partial x_{2}}+1 & \frac{\partial^{2} B}{\partial x_{2}^{2}}
\end{array}\right) \leq 0 .
\end{aligned}
$$

In fact, (4.4) is equivalent to (4.5).

Remark 9. If there is an extremal pair $u, v$ realizing the supremum in the definition of the Bellman function, then one of the matrices (4.5) must be degenerate. Indeed, assume that for some $u, v$ with averages $\langle u\rangle_{J}=x_{1},\langle v\rangle_{J}=x_{2}$ we have

$$
\mathbf{B}(x)=\frac{1}{|J|} \sum_{I \in \mathcal{I}} \alpha^{+} \alpha^{-}|I|\left|\Delta_{I} u\right|\left|\Delta_{I} v\right| .
$$


Then

$$
\begin{aligned}
\alpha^{+} \mathbf{B}\left(x^{+}\right)+\alpha^{-} \mathbf{B}\left(x^{-}\right)+\alpha^{+} \alpha^{-}\left|\Delta_{J} u\right|\left|\Delta_{J} v\right| \\
\geq \frac{\alpha^{+}}{\left|J^{+}\right|} \sum_{I \in \mathcal{I}^{+}} \alpha^{+} \alpha^{-}|I|\left|\Delta_{I} u\right|\left|\Delta_{I} v\right| \\
\quad+\frac{\alpha^{-}}{\left|J^{-}\right|} \sum_{I \in \mathcal{I}^{-}} \alpha^{+} \alpha^{-}|I|\left|\Delta_{I} u\right|\left|\Delta_{I} v\right|+\alpha^{+} \alpha^{-}\left|\Delta_{J} u\right|\left|\Delta_{J} v\right| \\
=\frac{1}{|J|} \sum_{I \in \mathcal{I}} \alpha^{+} \alpha^{-}|I|\left|\Delta_{I} u\right|\left|\Delta_{I} v\right|=\mathbf{B}(x) .
\end{aligned}
$$

Together with the assumption that $\mathbf{B}$ satisfies (4.3), this gives equality for some splitting of the initial interval $J$. If we assume that the infinitesimal equality in (4.4) is also true, then one of the matrices (4.5) must be degenerate. Equating the determinants of these matrices to zero, we get two versions of the Bellman equation:

$$
\frac{\partial^{2} B}{\partial x_{1}^{2}} \cdot \frac{\partial^{2} B}{\partial x_{1}^{2}}=\left(\frac{\partial^{2} B}{\partial x_{1} \partial x_{2}} \pm 1\right)^{2} .
$$

We show that our assumption about the infinitesimal equality in (4.4) can be proved in some situations. Fix a splitting for which equality occurs in (4.3), and suppose that the points $x^{+}$and $x^{-}$generated by this splitting are such that the entire straight line segment $L$ connecting the $x^{ \pm}$is in $\Omega$. Of course, $x \in L$. Take $\sigma=\operatorname{sgn} \Delta_{1} \Delta_{2}$ and consider the function

$$
\phi(x)=\mathbf{B}(x)+\sigma x_{1} x_{2} .
$$

Assumption (4.3) means exactly that $\phi$ is a concave function, because

$$
\alpha^{+} x_{1}^{+} x_{2}^{+}+\alpha^{-} x_{1}^{-} x_{2}^{-}=x_{1} x_{2}+\alpha^{+} \alpha^{-} \Delta_{1} \Delta_{2},
$$

which will be checked at the beginning of the proof of Lemma 4 . In particular, $\phi$ is concave on $L$. But in $(*)$ we have seen that

$$
\alpha^{+} \phi\left(x^{+}\right)+\alpha^{-} \phi\left(x^{-}\right) \geq \phi(x) .
$$

A concave function on an interval can satisfy the latter inequality only if it is linear on this interval. This means that one of the matrices (4.5) must be degenerate.

Reducing the Bellman equation to an ordinary differential equation. This can be done by using the function $\varphi$ introduced in Lemma 1. We shall look for a function $B$ of the form $B\left(x_{1}, x_{2}\right)=\varphi\left(x_{1} x_{2}\right)$. Then

$$
\frac{\partial B}{\partial x_{1}}=x_{2} \varphi^{\prime}, \quad \frac{\partial B}{\partial x_{2}}=x_{1} \varphi^{\prime}, \quad \frac{\partial^{2} B}{\partial x_{1}^{2}}=x_{2}^{2} \varphi^{\prime \prime}, \quad \frac{\partial^{2} B}{\partial x_{1} \partial x_{2}}=\varphi^{\prime}+x_{1} x_{2} \varphi^{\prime \prime}, \quad \frac{\partial^{2} B}{\partial x_{2}^{2}}=x_{1}^{2} \varphi^{\prime \prime} .
$$

Therefore, the matrix

$$
\left(\begin{array}{cc}
\frac{\partial^{2} B}{\partial x_{1}^{2}} & \frac{\partial^{2} B}{\partial x_{1} \partial x_{2}} \pm 1 \\
\frac{\partial^{2} B}{\partial x_{1} \partial x_{2}} \pm 1 & \frac{\partial^{2} B}{\partial x_{2}^{2}}
\end{array}\right)=\left(\begin{array}{cc}
x_{2}^{2} \varphi^{\prime \prime} & \varphi^{\prime}+x_{1} x_{2} \varphi^{\prime \prime} \pm 1 \\
\varphi^{\prime}+x_{1} x_{2} \varphi^{\prime \prime} \pm 1 & x_{1}^{2} \varphi^{\prime \prime}
\end{array}\right)
$$

is degenerate if and only if

$$
\begin{aligned}
\left(x_{2}^{2} \varphi^{\prime \prime}\right)\left(x_{1}^{2} \varphi^{\prime \prime}\right)-\left(\varphi^{\prime}+x_{1} x_{2} \varphi^{\prime \prime} \pm 1\right)^{2} & =0, \\
s^{2}\left(\varphi^{\prime \prime}\right)^{2}-\left(\varphi^{\prime}+s \varphi^{\prime \prime} \pm 1\right)^{2} & =0, \\
\left(\varphi^{\prime} \pm 1\right)\left(\varphi^{\prime}+2 s \varphi^{\prime \prime} \pm 1\right) & =0 .
\end{aligned}
$$

We immediately see that no solution of the equation $\varphi^{\prime} \pm 1=0$ can suit us, because then one of the matrices in (4.5) is identically zero and the second turns into the matrix $\left(\begin{array}{cc}0 & \pm 2 \\ \pm 2 & 0\end{array}\right)$, which is not negative definite. 
Solution of the ordinary differential equation. So, we need to find a solution of one of the differential equations

$$
2 s \varphi^{\prime \prime}+\varphi^{\prime} \pm 1=0
$$

such that conditions (4.5), or (in terms of $\varphi$ )

$$
\left(\begin{array}{cc}
x_{2}^{2} \varphi^{\prime \prime} & \varphi^{\prime}+x_{1} x_{2} \varphi^{\prime \prime} \pm 1 \\
\varphi^{\prime}+x_{1} x_{2} \varphi^{\prime \prime} \pm 1 & x_{1}^{2} \varphi^{\prime \prime}
\end{array}\right) \leq 0,
$$

are fulfilled. Note that these conditions must be fulfilled for both signs, whatever sign was taken in equation (4.7). Dividing this equation by $2 \sqrt{s}$, we get

$$
\begin{aligned}
\sqrt{s} \varphi^{\prime \prime}+\frac{1}{2 \sqrt{s}}\left(\varphi^{\prime} \pm 1\right) & =0 \\
{\left[\sqrt{s}\left(\varphi^{\prime} \pm 1\right)\right]^{\prime} } & =0 \\
\varphi^{\prime} \pm 1 & =\frac{C_{ \pm}}{2 \sqrt{s}}, \\
\varphi \pm s & =C_{ \pm} \sqrt{s}+\text { const. }
\end{aligned}
$$

The value of the integration constant can be found from the boundary condition $\varphi\left(m^{2}\right)=$ 0 :

$$
\varphi(s)=\mp\left(s-m^{2}\right)+C_{ \pm}(\sqrt{s}-m) .
$$

We rewrite conditions (4.8) in a scalar form:

$$
\varphi^{\prime \prime} \leq 0, \quad\left(s \varphi^{\prime \prime}\right)^{2} \geq\left(s \varphi^{\prime \prime}+\varphi^{\prime} \pm 1\right)^{2} .
$$

For our solutions the inequality $\varphi^{\prime \prime} \leq 0$ is equivalent to $C_{ \pm} \geq 0$. We check that the second condition is not fulfilled for the solution $\varphi=s-m^{2}+C_{-}(\sqrt{s}-m)$ :

$$
s \varphi^{\prime \prime}+\varphi^{\prime}+1=s\left(-\frac{C_{-}}{4 s^{3 / 2}}\right)+\left(1+\frac{C_{-}}{2 s^{1 / 2}}\right)+1=\frac{C_{-}}{4 \sqrt{s}}+2>\frac{C_{-}}{4 \sqrt{s}}=\left|s \varphi^{\prime \prime}\right| .
$$

It remains to consider the solution

$$
\varphi(s)=-s+m^{2}+C_{+}(\sqrt{s}-m) .
$$

We have

$$
\begin{gathered}
\varphi^{\prime}(s)=-1+\frac{C_{+}}{2 s^{1 / 2}}, \quad \varphi^{\prime \prime}(s)=-\frac{C_{+}}{4 s^{3 / 2}}, \\
s \varphi^{\prime \prime}+\varphi^{\prime}+1=\frac{C_{+}}{4 s^{1 / 2}}=\left|s \varphi^{\prime \prime}\right|, \\
s \varphi^{\prime \prime}+\varphi^{\prime}-1=\frac{C_{+}}{4 s^{1 / 2}}-2, \\
\left(s \varphi^{\prime \prime}\right)^{2} \geq\left(s \varphi^{\prime \prime}+\varphi^{\prime} \pm 1\right)^{2} \Longleftrightarrow \frac{C_{+}^{2}}{16 s} \geq\left(\frac{C_{+}}{4 s^{1 / 2}}-2\right)^{2} \Longleftrightarrow C_{+} \geq 4 \sqrt{s} .
\end{gathered}
$$

Since the last-written inequality must be fulfilled for all $s \in\left[\mathrm{m}^{2}, M^{2}\right]$, we get the restriction

$$
C_{+} \geq 4 M
$$

We shall check that the minimal possible value of the constant $C_{+}=4 M$ gives us the true Bellman function. 


\section{$\S 5$. The proofs of Theorem 2 and Corollary to Theorem 3}

For a while we forget about the candidate found for the role of the Bellman function and prove the following general assertion.

Lemma 3. Let $x^{ \pm}$be a pair of arbitrary points in $\Omega$, and let $\alpha^{ \pm}$be any pair of splitting numbers $\left(\alpha^{ \pm} \geq 0, \alpha^{+}+\alpha^{-}=1\right)$ such that the point $x \stackrel{\text { def }}{=} \alpha^{+} x^{+}+\alpha^{-} x^{-}$is in $\Omega$. If a function $B$ defined on $\Omega$ satisfies inequality (4.3) for all such triples $\left(x^{+}, x^{-}, x\right)$, then

$$
\mathbf{B}(x ; m, M) \leq B(x ; m, M) \quad \text { and } \quad \mathbf{B}_{\mathcal{D}}(x ; m, M) \leq B(x ; m, M) .
$$

Proof. Fix an arbitrary partition $\mathcal{I}$ of $J$ (in the dyadic case $\mathcal{I}=\mathcal{D}$ ) and any pair $u, v$ of admissible functions (i.e., satisfying condition (2.1) for all $I \subset J$ in the nondyadic case and for all $I \in \mathcal{D}$ in the dyadic case).

We rewrite inequality (4.3) for $I=I_{n, m}$ in the following form:

$$
\begin{aligned}
& \alpha_{I}^{+} \alpha_{I}^{-}\left|\langle u\rangle_{I^{+}}-\langle u\rangle_{I^{-}}\right|\left|\langle v\rangle_{I^{+}}-\langle v\rangle_{I^{-}}\right| \\
& \quad=\alpha_{n+1,2 m} \alpha_{n+1,2 m+1}\left|x_{1}^{n+1,2 m}-x_{1}^{n+1,2 m+1}\right|\left|x_{2}^{n+1,2 m}-x_{2}^{n+1,2 m+1}\right| \\
& \quad \leq B\left(x^{n, m}\right)-\alpha_{n+1,2 m} B\left(x^{n+1,2 m}\right)-\alpha_{n+1,2 m+1} B\left(x^{n+1,2 m+1}\right),
\end{aligned}
$$

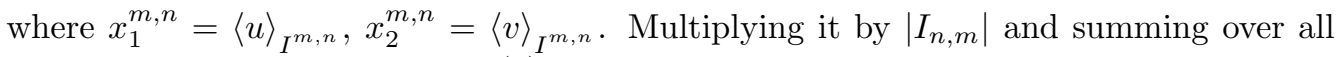
intervals of the $n$th generation $\mathcal{I}^{(n)}$, we get

$$
\begin{aligned}
\sum_{m=0}^{2^{n}-1} & \alpha_{n+1,2 m} \alpha_{n+1,2 m+1}\left|x_{1}^{n+1,2 m}-x_{1}^{n+1,2 m+1}\right|\left|x_{2}^{n+1,2 m}-x_{2}^{n+1,2 m+1}\right|\left|I_{n, m}\right| \\
& \leq \sum_{m=0}^{2^{n}-1}\left[B\left(x^{n, m}\right)\left|I_{n, m}\right|-B\left(x^{n+1,2 m}\right)\left|I_{n+1,2 m}\right|-B\left(x^{n+1,2 m+1}\right)\left|I_{n+1,2 m+1}\right|\right] \\
& =\sum_{m=0}^{2^{n}-1} B\left(x^{n, m}\right)\left|I_{n, m}\right|-\sum_{m=0}^{2^{n+1}-1} B\left(x^{n+1, m}\right)\left|I_{n+1, m}\right| \\
& =\sum_{I \in \mathcal{I}^{(n)}} B\left(\langle u\rangle_{I},\langle v\rangle_{I}\right)|I|-\sum_{I \in \mathcal{I}^{(n+1)}} B\left(\langle u\rangle_{I},\langle v\rangle_{I}\right)|I| .
\end{aligned}
$$

Now, we sum over $n$ to obtain

$$
\begin{aligned}
& \sum_{n=0}^{N} \sum_{I \in \mathcal{I}^{(n)}} \alpha_{I}^{+} \alpha_{I}^{-}\left|\langle u\rangle_{I^{+}}-\langle u\rangle_{I^{-}}\right|\left|\langle v\rangle_{I^{+}}-\langle v\rangle_{I^{-}}\right||I| \\
& \quad \leq B\left(\langle u\rangle_{J},\langle v\rangle_{J}\right)|J|-\sum_{I \in \mathcal{I}^{(n+1)}} B\left(\langle u\rangle_{I},\langle v\rangle_{I}\right)|I| \\
& \quad \leq B\left(\langle u\rangle_{J},\langle v\rangle_{J}\right)|J| .
\end{aligned}
$$

Passing to the limit as $N \rightarrow \infty$ and taking the supremum over all admissible pair of functions $u, v$ with $\langle u\rangle_{J}=x_{1},\langle v\rangle_{J}=x_{2}$, we arrive at the assertion of the lemma for $\mathbf{B}_{\mathcal{D}}(x)$. To get the desired inequality in the nondyadic case, it remains to take yet another supremum over all possible partitions $\mathcal{I}$.

From now on, we denote by the symbol $\mathcal{B}$ the candidate for the Bellman function we have found, i.e.,

$$
\mathcal{B}(x ; m, M)=\varphi\left(x_{1} x_{2} ; m, M\right)=4 M \sqrt{x_{1} x_{2}}-x_{1} x_{2}+m^{2}-4 m M .
$$

We are going to check that the assumptions of Lemma 3 are fulfilled for this function. 
Lemma 4. Let $x^{ \pm}$be a pair of arbitrary points in $\Omega$, and let $\alpha^{ \pm}$be any pair of splitting numbers $\left(\alpha^{ \pm} \geq 0, \alpha^{+}+\alpha^{-}=1\right)$ such that the point $x \stackrel{\text { def }}{=} \alpha^{+} x^{+}+\alpha^{-} x^{-}$is in $\Omega$. Then the function $\mathcal{B}$ satisfies inequality (4.3).

Proof. As before, we put $\Delta_{i}=x_{i}^{+}-x_{i}^{-}$and check the relation $\alpha^{+} x_{1}^{+} x_{2}^{+}+\alpha^{-} x_{1}^{-} x_{2}^{-}=$ $x_{1} x_{2}+\alpha^{+} \alpha^{-} \Delta_{1} \Delta_{2}$ :

$$
\begin{aligned}
& \alpha^{+} x_{1}^{+} x_{2}^{+}+\alpha^{-} x_{1}^{-} x_{2}^{-} \\
& \quad=\alpha^{+}\left(x_{1}+\alpha^{-} \Delta_{1}\right)\left(x_{2}+\alpha^{-} \Delta_{2}\right)+\alpha^{-}\left(x_{1}-\alpha^{+} \Delta_{1}\right)\left(x_{2}-\alpha^{+} \Delta_{2}\right) \\
& \quad=\left(\alpha^{+}+\alpha^{-}\right) x_{1} x_{2}+\left(\alpha^{+}\left(\alpha^{-}\right)^{2}+\left(\alpha^{+}\right)^{2} \alpha^{-}\right) \Delta_{1} \Delta_{2} \\
& \quad=x_{1} x_{2}+\alpha^{+} \alpha^{-} \Delta_{1} \Delta_{2} .
\end{aligned}
$$

Therefore,

$$
\begin{aligned}
& \mathcal{B}(x)-\alpha^{+} \mathcal{B}\left(x^{+}\right)-\alpha^{-} \mathcal{B}\left(x^{-}\right) \\
& =4 M\left[\sqrt{x_{1} x_{2}}-\alpha^{+} \sqrt{x_{1}^{+} x_{2}^{+}}-\alpha^{-} \sqrt{x_{1}^{-} x_{2}^{-}}\right]-\left[x_{1} x_{2}-\alpha^{+} x_{1}^{+} x_{2}^{+}-\alpha^{-} x_{1}^{-} x_{2}^{-}\right] \\
& =4 M\left[\sqrt{x_{1} x_{2}}-\alpha^{+} \sqrt{x_{1}^{+} x_{2}^{+}}-\alpha^{-} \sqrt{x_{1}^{-} x_{2}^{-}}\right]+\alpha^{+} \alpha^{-} \Delta_{1} \Delta_{2} .
\end{aligned}
$$

If $\Delta_{1} \Delta_{2} \geq 0$, then it suffices to refer to the concavity of the function $x \mapsto \sqrt{x_{1} x_{2}}$ in the first quadrant:

$$
d^{2}\left(\sqrt{x_{1} x_{2}}\right)=\left(\begin{array}{cc}
-\frac{1}{4} \sqrt{\frac{x_{2}}{x_{1}^{3}}} & \frac{1}{4 \sqrt{x_{1} x_{2}}} \\
\frac{1}{4 \sqrt{x_{1} x_{2}}} & -\frac{1}{4} \sqrt{\frac{x_{1}}{x_{2}^{3}}}
\end{array}\right) \leq 0,
$$

whence

$$
\sqrt{x_{1} x_{2}}-\alpha^{+} \sqrt{x_{1}^{+} x_{2}^{+}}-\alpha^{-} \sqrt{x_{1}^{-} x_{2}^{-}} \geq 0
$$

and

$$
\mathcal{B}(x)-\alpha^{+} \mathcal{B}\left(x^{+}\right)-\alpha^{-} \mathcal{B}\left(x^{-}\right) \geq \alpha^{+} \alpha^{-} \Delta_{1} \Delta_{2} .
$$

If $\Delta_{1} \Delta_{2}<0$, we use the concavity of the function $t \mapsto \sqrt{t}$ (indeed, $4(\sqrt{t})^{\prime \prime}=-t^{-3 / 2}<0$ ):

$$
\begin{aligned}
\sqrt{x_{1} x_{2}} & -\alpha^{+} \sqrt{x_{1}^{+} x_{2}^{+}}-\alpha^{-} \sqrt{x_{1}^{-} x_{2}^{-}} \\
& \geq \sqrt{x_{1} x_{2}}-\sqrt{\alpha^{+} x_{1}^{+} x_{2}^{+}+\alpha^{-} x_{1}^{-} x_{2}^{-}} \\
& =\sqrt{x_{1} x_{2}}-\sqrt{x_{1} x_{2}+\alpha^{+} \alpha^{-} \Delta_{1} \Delta_{2}} \\
& =-\frac{\alpha^{+} \alpha^{-} \Delta_{1} \Delta_{2}}{\sqrt{x_{1} x_{2}}+\sqrt{x_{1} x_{2}+\alpha^{+} \alpha^{-} \Delta_{1} \Delta_{2}}} \\
& \geq-\frac{\alpha^{+} \alpha^{-} \Delta_{1} \Delta_{2}}{2 \sqrt{x_{1} x_{2}}} .
\end{aligned}
$$

Therefore,

$$
\begin{aligned}
\mathcal{B}(x) & -\alpha^{+} \mathcal{B}\left(x^{+}\right)-\alpha^{-} \mathcal{B}\left(x^{-}\right) \\
& \geq 4 M\left(-\frac{\alpha^{+} \alpha^{-} \Delta_{1} \Delta_{2}}{2 \sqrt{x_{1} x_{2}}}\right)+\alpha^{+} \alpha^{-} \Delta_{1} \Delta_{2}=\alpha^{+} \alpha^{-}\left|\Delta_{1} \Delta_{2}\right|\left(\frac{2 M}{\sqrt{x_{1} x_{2}}}-1\right) \\
& \geq \alpha^{+} \alpha^{-}\left|\Delta_{1} \Delta_{2}\right| .
\end{aligned}
$$

Combined with the preceding lemma, Lemma 4 proves Theorem 2 and the Corollary to Theorem 3. As you see, this proof is very short and does not require the considerations of the preceding section if somehow we know the Bellman function a priori. 
The rest of the paper is devoted to the proof of Theorem 3, which means constructing an extremal pair of functions (in fact, an approximating sequence), which gives us the reverse inequalities

$$
\mathbf{B}(x ; m, M) \geq \mathcal{B}(x ; m, M) \quad \text { and } \quad \mathbf{B}_{\mathcal{D}}(x ; m, M) \geq \mathcal{B}(x ; m, M) .
$$

\section{$\S 6$. HOW TO FIND AN EXTREMAL FUNCTION}

The reader who is interested in the formal proof rather than in underlying ideas may skip this section, as was the case with $\S 4$ : here again we shall try to explain how we arrive at the construction that will be presented formally in the next section.

If we look for a pair $u, v$ that realizes the supremum in the definition of $\mathbf{B}$, we need to have equality in the limit in (5.2), and therefore, we need to have equality in each term of the sum (5.1). So, at each step we must perform a splitting such that the corresponding differences $\Delta=x^{+}-x^{-}$are in the kernel of one of the matrices (4.5). For this reason, we shall investigate the vector field of the kernel vectors of these matrices for our candidate $\mathcal{B}$ for the role of the Bellman function. If we substitute $\varphi\left(x_{1} x_{2}\right)=$ $-x_{1} x_{2}+m^{2}+4 M\left(\sqrt{x_{1} x_{2}}-m\right)$ in formula (4.8), which represents the matrices (4.5) in terms of $\varphi$, we arrive at the following expressions:

$$
\left(\begin{array}{cc}
-M \sqrt{\frac{x_{2}}{x_{1}^{3}}} & \frac{M}{\sqrt{x_{1} x_{2}}}-1 \pm 1 \\
\frac{M}{\sqrt{x_{1} x_{2}}}-1 \pm 1 & -M \sqrt{\frac{x_{1}}{x_{2}^{3}}}
\end{array}\right) .
$$

For the "+" sign we get a degenerate matrix at every point $x$ :

$$
\left(\begin{array}{cc}
-M \sqrt{\frac{x_{2}}{x_{1}}} & \frac{M}{\sqrt{x_{1} x_{2}}} \\
\frac{M}{\sqrt{x_{1} x_{2}}} & -M \sqrt{\frac{x_{1}}{x_{2}}}
\end{array}\right),
$$

with the kernel vector $\Delta=\left(\begin{array}{l}x_{1} \\ x_{2}\end{array}\right)$ directed along the ray starting at the origin. This means that our basic inequality (4.3) turns into equality up to terms of the second order with respect to the length of $\Delta$ if the points $x, x^{ \pm}$lie on that ray. We show that equality occurs not only infinitesimally, but it is in fact a strict identity for all such triples of points. Take an arbitrary point $x=\left(x_{1}, x_{2}\right), \Delta=\left(\Delta_{1}, \Delta_{2}\right)=\left(x_{1}, x_{2}\right) t, x^{+}=x+\alpha^{-} \Delta=\left(1+\alpha^{-} t\right) x$, and $x^{-}=x-\alpha^{+} \Delta=\left(1-\alpha^{+} t\right) x$. Then

$$
\mathcal{B}\left(x^{ \pm}\right)=4 M\left(1 \pm \alpha^{\mp} t\right) \sqrt{x_{1} x_{2}}-\left(1 \pm \alpha^{\mp} t\right)^{2} x_{1} x_{2}+m^{2}-4 m M,
$$

whence

$$
\begin{aligned}
& \alpha^{+} \mathcal{B}\left(x_{+}\right)+\alpha^{-} \mathcal{B}\left(x_{-}\right) \\
& \quad=4 M \sqrt{x_{1} x_{2}}-\left(1+\alpha^{+} \alpha^{-} t^{2}\right) x_{1} x_{2}+m^{2}-4 m M \\
& \quad=\mathcal{B}(x)-\alpha^{+} \alpha^{-} t^{2} x_{1} x_{2}=\mathcal{B}(x)-\alpha^{+} \alpha^{-}\left|\Delta_{1} \Delta_{2}\right| .
\end{aligned}
$$

Remark 10. So, we know how to find an extremal pair $u, v$ for an arbitrary point $x$ in the interior of $\Omega$ : we must take the points $x^{ \pm}$at which the ray starting at the origin and passing through $x$ intersects the boundary of $\Omega$. We take $x^{-}$(say) on the lower boundary, i.e., $x_{1}^{-}=m \sqrt{x_{1} / x_{2}}, x_{2}^{-}=m \sqrt{x_{2} / x_{1}}$, and $x^{+}$on the upper boundary, i.e., $x_{1}^{+}=M \sqrt{x_{1} / x_{2}}, x_{2}^{+}=M \sqrt{x_{2} / x_{1}}$. Then we define on $J^{-}$the extremal pair corresponding to the point of the lower boundary, i.e., the constant functions $u=x_{1}^{-}$, $v=x_{2}^{-}$, and on $J^{+}$the (unknown) extremal pair corresponding to the point of the upper boundary. That is, the problem is to construct the extremal pair for the points of the upper boundary.

The procedure should be changed a little in the dyadic case, when we are not free to choose the splitting coefficient and at every step we split our interval into two equal 
parts. In this situation we can take a rearrangement of the pair constructed. On $J^{-}$, we leave the constant function as it was, but we represent the interval $J^{+}$as a union of dyadic intervals and on every such interval we define the pair $u, v$ to be the suitably scaled pair corresponding to the point $x^{+}$of the upper boundary.

In the nondyadic case we still need to check that this procedure provides an admissible pair (i.e., condition (2.1) is fulfilled for every subinterval $I \subset J$ ), whereas in the dyadic case the resulting pair is admissible automatically (i.e., condition (2.1) is fulfilled for every dyadic subinterval $I \subset J)$. Indeed, the average over any dyadic interval $I$ is a convex combination of the averages over $I \cap J^{-}$(which are the coordinates of the point $x^{-}$) and the averages over $I \cap J^{+}$(which are the coordinates of the point $x^{+}$, because $I \cap J^{+}$is a union of dyadic intervals with the property that the averages over each of them are the same, namely, the coordinates of the point $x^{+}$). Therefore, these averages give us a point on the straight line segment with the endpoints $x^{ \pm}$, which is inside the domain $\Omega$.

Returning to the extremal trajectories found, we see that they cannot help us to construct the extremal pair for the points of the upper boundary, because we cannot represent a point of the upper boundary as a convex combination of two points $x^{ \pm}$lying on the ray passing through the origin and different from $x$ itself. But here the second matrix in 6.1 with the "-" sign can help us.

Indeed, this matrix is nonsingular everywhere in $\Omega$ except the upper boundary. For $x_{1} x_{2}=M^{2}$ this matrix is

$$
\left(\begin{array}{cc}
-\frac{x_{2}}{x_{1}} & -1 \\
-1 & -\frac{x_{1}}{x_{2}}
\end{array}\right)
$$

and its kernel vector $\Delta=\left(\begin{array}{c}x_{1} \\ -x_{2}\end{array}\right)$ is tangent to the upper boundary. Therefore, the upper boundary itself is an "extremal trajectory" and we can "split" the points on it in the tangent direction. However, for any finite $\Delta$ we have strict inequality in (4.3), but the difference between the left-hand side and the right-hand side is of the fourth order in length of $\Delta$. For this reason, we cannot get an extremal pair $u, v$, but if we split the intervals corresponding to the points on the upper boundary in such a way that the averages on the left and right subintervals become closer and closer, we get a sequence of functions realizing the supremum in the definition of the Bellman function.

\section{§7. Construction of an extremal sequence}

To prove the reverse inequality, we need to construct a sequence of test functions $u_{n}, v_{n} \in L^{1}(J)$ satisfying the conditions $m^{2} \leq\left\langle u_{n}\right\rangle_{I}\left\langle v_{n}\right\rangle_{I} \leq M^{2},\left\langle u_{n}\right\rangle_{J}=x_{1},\left\langle v_{n}\right\rangle_{J}=$ $x_{2}$, and such that the corresponding sums in the definition of $\mathbf{B}$ tend to $\mathbf{B}\left(x_{1}, x_{2}\right)$.

For this, we fix a constant $C, m<C \leq M$, an integer $N$, and the larger root $a(a>1)$ of the equation

$$
2^{N}=\frac{C-m}{C} \cdot \frac{a^{2}+1}{(a-1)^{2}},
$$

i.e.,

$$
a=\frac{2^{N} C+\sqrt{(C-m)\left(2^{N+1} C-C+m\right)}}{2^{N} C-C+m} .
$$


Take $J=[0,1]$ and define the following sequence of step functions $u_{n}(t)=u_{n}(t ; N, m, C)$, $v_{n}(t)=v_{n}(t ; N, m, C)$ on $[0,1]: u_{0}(t)=v_{0}(t)=C$,

$$
u_{n+1}(t)=\left\{\begin{array}{lr}
\frac{m}{a}, & 0 \leq t<2^{-N-1}, \\
\frac{1}{a} u_{n}\left(2^{k+1} t-1\right), & 2^{-k-1} \leq t<2^{-k}, \\
a u_{n}\left(2-2^{k+1}(1-t)\right), & 1-2^{-k}<t \leq 1-2^{-k-1}, \\
a m, & 1-2^{-N-1}<t \leq 1,
\end{array}\right.
$$

and

$$
v_{n+1}(t)=\left\{\begin{array}{lr}
a m, & 0 \leq t<2^{-N-1}, \\
a v_{n}\left(2^{k+1} t-1\right), & 2^{-k-1} \leq t<2^{-k}, \\
\frac{1}{a} v_{n}\left(2-2^{k+1}(1-t)\right), & 1-2^{-k}<t \leq 1-2^{-k-1}, \\
\frac{m}{a}, & 1-2^{-N-1}<t \leq 1 .
\end{array}\right.
$$

In these formulas $k$ runs from 1 to $N$.

Lemma 5. For all $n \geq 0$, we have

$$
\begin{gathered}
v_{n}(t)=u_{n}(1-t), \\
\left\langle u_{n}\right\rangle_{[0,1]}=\left\langle v_{n}\right\rangle_{[0,1]}=C, \\
\left\langle u_{n+1}\right\rangle_{\left[2^{-k-1}, 2^{-k}\right]}=\frac{C}{a}, \\
\left\langle v_{n+1}\right\rangle_{\left[2^{-k-1}, 2^{-k}\right]}=a C, \quad 1 \leq k \leq N, \\
\left\langle u_{n+1}\right\rangle_{\left[0, \frac{1}{2}\right]}=\left\langle v_{n+1}\right\rangle_{\left[\frac{1}{2}, 1\right]}=\frac{2 C}{1+a^{2}}, \\
\left\langle u_{n+1}\right\rangle_{\left[\frac{1}{2}, 1\right]}=\left\langle v_{n+1}\right\rangle_{\left[0, \frac{1}{2}\right]}=\frac{2 a^{2} C}{1+a^{2}} .
\end{gathered}
$$

Proof. We check relation (7.4) by induction. For $n=0$, we have $v_{0}(t)=C=u_{0}(1-t)$. We verify identity (7.4), say, for $2^{-k-1} \leq t<2^{-k}$ :

$$
\begin{aligned}
v_{n+1}(t) & =a v_{n}\left(2^{k+1} t-1\right)=a u_{n}\left(2-2^{k+1} t\right) \\
& =u_{n+1}(1-t) .
\end{aligned}
$$

By the definition (7.2)-(17.3), relation (7.5) implies (7.6). Now, using (7.6) together with (7.1), we calculate averages over the interval $\left[0, \frac{1}{2}\right]$ :

$$
\begin{aligned}
\left\langle u_{n+1}\right\rangle_{\left[0, \frac{1}{2}\right]} & =2 \int_{0}^{\frac{1}{2}} u_{n+1}(t) d t=2^{-N} \frac{m}{a}+\left(1-2^{-N}\right) \frac{C}{a}=\frac{C}{a}-2^{-N} \frac{C-m}{a} \\
& =\frac{C}{a}-\frac{C}{C-m} \cdot \frac{(a-1)^{2}}{a^{2}+1} \cdot \frac{C-m}{a}=\frac{2 C}{a^{2}+1}, \\
\left\langle v_{n+1}\right\rangle_{\left[0, \frac{1}{2}\right]} & =2 \int_{0}^{\frac{1}{2}} v_{n+1}(t) d t=2^{-N} a m+\left(1-2^{-N}\right) a C=a C-2^{-N} a(C-m) \\
& =a C-\frac{C}{C-m} \cdot \frac{(a-1)^{2}}{a^{2}+1} a(C-m)=\frac{2 a^{2} C}{a^{2}+1} .
\end{aligned}
$$

Together with (7.4), this proves (7.7).

Adding the identities (7.7), we get (7.5) for the next value of the index $n$. Since (7.5) is clearly true for $n=0$, this completes the proof of the lemma by induction. 
Now we calculate the sum in the definition of the Bellman function:

$$
b_{n}(N, m, C)=\frac{1}{4} \sum_{I \in \mathcal{D}}|I|\left|\left\langle u_{n}\right\rangle_{I^{+}}-\left\langle u_{n}\right\rangle_{I^{-}}\right|\left|\left\langle v_{n}\right\rangle_{I^{+}}-\left\langle v_{n}\right\rangle_{I^{-}}\right| .
$$

\section{Lemma 6.}

$$
b_{n}(N, m, C)=(C-m)\left(3 C-m-(C-m) 2^{-N+1}\right)\left(1-\left(1-2^{-N}\right)^{n}\right) .
$$

Proof. We proceed by induction on $n$. For $n=0$, we have $u_{0}(t)=v_{0}(t)=C$ and $b_{0}=0$. To calculate $b_{n+1}$ (assuming that the formula for $b_{n}$ is true), we split the set of all dyadic intervals $\mathcal{D}$ into three groups:

$\mathcal{D}_{1}$ consists of all dyadic intervals inside of the interval $\left[2^{-N-1}, 1-2^{-N-1}\right]$,

$\mathcal{D}_{2}=\left\{\left[0,2^{-k}\right]: 1 \leq k \leq N\right\} \cup\left\{\left[1-2^{-k}, 1\right]: 1 \leq k \leq N\right\}$,

$\mathcal{D}_{3}$ consists of all other dyadic intervals.

To calculate the sum over $\mathcal{D}_{1}$, we use the inductive definitions of $u_{n+1}$ and $v_{n+1}$ together with the inductive hypothesis

$$
\begin{aligned}
\frac{1}{4} & \sum_{I \in \mathcal{D}_{1}}|I|\left|\left\langle u_{n+1}\right\rangle_{I^{+}}-\left\langle u_{n+1}\right\rangle_{I^{-}}\right|\left|\left\langle v_{n+1}\right\rangle_{I^{+}}-\left\langle v_{n+1}\right\rangle_{I^{-}}\right| \\
& =\left(1-2^{-N}\right) b_{n} \\
& =(C-m)\left(3 C-m-(C-m) 2^{-N+1}\right)\left(1-2^{-N}-\left(1-2^{-N}\right)^{n+1}\right) .
\end{aligned}
$$

By Lemma 5 and relations (7.2) $-(7.3)$, we have

$$
\left\langle u_{n+1}\right\rangle_{\left[2^{-k-1}, 2^{-k}\right]}=\frac{C}{a}, \quad\left\langle v_{n+1}\right\rangle_{\left[2^{-k-1}, 2^{-k}\right]}=a C, \quad 1 \leq k \leq N,
$$

and

whence

$$
\left\langle u_{n+1}\right\rangle_{\left[0,2^{-N-1}\right]}=\frac{m}{a}, \quad\left\langle v_{n+1}\right\rangle_{\left[0,2^{-N-1}\right]}=a m,
$$

$$
\begin{aligned}
\left\langle u_{n+1}\right\rangle_{\left[0,2^{-k}\right]} & =\frac{2^{-N-1} \frac{m}{a}+\left[2^{-k}-2^{-N-1}\right] \frac{C}{a}}{2^{-k}} \\
& =\frac{C}{a}-2^{k-N-1} \frac{C-m}{a}, \\
\left\langle v_{n+1}\right\rangle_{\left[0,2^{-k}\right]} & =\frac{2^{-N-1} a m+\left[2^{-k}-2^{-N-1}\right] a C}{2^{-k}} \\
& =a C-2^{k-N-1} a(C-m) .
\end{aligned}
$$

By (7.4), the intervals $\left[0,2^{-k}\right]$ and $\left[1-2^{-k}, 1\right]$ give equal terms; therefore,

$$
\begin{aligned}
& \frac{1}{4} \sum_{I \in \mathcal{D}_{2}}|I|\left|\left\langle u_{n+1}\right\rangle_{I^{+}}-\left\langle u_{n+1}\right\rangle_{I^{-}}\right|\left|\left\langle v_{n+1}\right\rangle_{I^{+}}-\left\langle v_{n+1}\right\rangle_{I^{-}}\right| \\
& \quad=\frac{1}{2} \sum_{k=1}^{N} 2^{-k}\left|\left\langle u_{n+1}\right\rangle_{\left[0,2^{-k-1}\right]}-\left\langle u_{n+1}\right\rangle_{\left[2^{-k-1}, 2^{-k}\right]}\right|\left|\left\langle v_{n+1}\right\rangle_{\left[0,2^{-k-1}\right]}-\left\langle v_{n+1}\right\rangle_{\left[2^{-k-1}, 2^{-k}\right]}\right| \\
& \quad=\frac{1}{2} \sum_{k=1}^{N} 2^{-k} \cdot 2^{k-N} \frac{C-m}{a} \cdot 2^{k-N} a(C-m) \\
& \quad=(C-m)^{2} 2^{-2 N-1} \sum_{k=1}^{N} 2^{k}=(C-m)^{2} 2^{-N}\left(1-2^{-N}\right)
\end{aligned}
$$


To prepare further calculations, we note that (7.1) implies that

$$
\begin{aligned}
\frac{(a-1)^{2}}{a^{2}+1} & =\frac{C-m}{C} 2^{-N}, \\
\frac{2 a}{a^{2}+1} & =1-\frac{C-m}{C} 2^{-N},
\end{aligned}
$$

and therefore,

$$
\begin{aligned}
\left(\frac{a^{2}-1}{a^{2}+1}\right)^{2} & =1-\left(\frac{2 a}{a^{2}+1}\right)^{2}=1-\left(1-\frac{C-m}{C} 2^{-N}\right)^{2} \\
& =\left(2-\frac{C-m}{C} 2^{-N}\right) \frac{C-m}{C} 2^{-N} .
\end{aligned}
$$

Finally, we note that in $\mathcal{D}_{3}$ only the interval $I=[0,1]$ gives a nonzero term, because for all other $I \in \mathcal{D}_{3}$ we have $I \subset\left[0,2^{-N-1}\right] \cup\left[1-2^{-N-1}, 1\right]$, where the functions $u_{n}, v_{n}$ are constant. So, using (17.7) and (7.9) we get

$$
\begin{aligned}
& \frac{1}{4} \sum_{I \in \mathcal{D}_{3}}|I|\left|\left\langle u_{n+1}\right\rangle_{I^{+}}-\left\langle u_{n+1}\right\rangle_{I^{-}}\right|\left|\left\langle v_{n+1}\right\rangle_{I^{+}}-\left\langle v_{n+1}\right\rangle_{I^{-}}\right| \\
& =\frac{1}{4}\left|\left\langle u_{n+1}\right\rangle_{\left[\frac{1}{2}, 1\right]}-\left\langle u_{n+1}\right\rangle_{\left[0, \frac{1}{2}\right]}\right|\left|\left\langle v_{n+1}\right\rangle_{\left[\frac{1}{2}, 1\right]}-\left\langle v_{n+1}\right\rangle_{\left[0, \frac{1}{2}\right]}\right| \\
& =C^{2}\left(\frac{a^{2}-1}{a^{2}+1}\right)^{2}=(C-m) 2^{-N}\left(2 C-2^{-N}(C-m)\right) .
\end{aligned}
$$

Collecting all three sums, we get

$$
\begin{aligned}
b_{n+1}= & (C-m)\left(3 C-m-(C-m) 2^{-N+1}\right)\left(1-2^{-N}-\left(1-2^{-N}\right)^{n+1}\right) \\
& +(C-m)^{2} 2^{-N}\left(1-2^{-N}\right)+(C-m) 2^{-N}\left(2 C-2^{-N}(C-m)\right) \\
= & (C-m)\left(3 C-m-(C-m) 2^{-N+1}\right)\left(1-\left(1-2^{-N}\right)^{n+1}\right) \\
& -(C-m) 2^{-N}\left(3 C-m-(C-m) 2^{-N+1}-(C-m)\left(1-2^{-N}\right)-2 C+2^{-N}(C-m)\right) \\
= & (C-m)\left(3 C-m-(C-m) 2^{-N+1}\right)\left(1-\left(1-2^{-N}\right)^{n+1}\right) .
\end{aligned}
$$

The following lemma, combined with Lemma 3, completes the proof the theorem for the Bellman function $\mathbf{B}_{\mathcal{D}}$.

\section{Lemma 7.}

$$
\mathbf{B}_{\mathcal{D}}(x ; m, M) \geq \mathcal{B}(x ; m, M) .
$$

Proof. We fix a point $x$ on the upper boundary of $\Omega_{m, M}$, i.e., $x_{1} x_{2}=M^{2}$. Using the families $u_{n}(t ; N, m, C), v_{n}(t ; N, m, C)$ constructed above, we consider the following functions:

$$
u(t)=\frac{x_{1}}{M} u_{n}(t ; N, m, M) \quad \text { and } \quad v(t)=\frac{x_{2}}{M} v_{n}(t ; N, m, M) .
$$

By Lemma 5, we have $\langle u\rangle_{[0,1]}=x_{1},\langle v\rangle_{[0,1]}=x_{2}$. In order to employ these functions as test functions for estimating $\mathbf{B}_{\mathcal{D}}(x)$ from below, we only need to check that $u$ and $v$ satisfy condition (2.1) for all dyadic intervals $I$. Since $u(t) v(t)=u_{n}(t ; N, m, M) v_{n}(t ; N, m, M) \geq$ $m^{2}$ (in fact, the latter product has only two values: $m^{2}$ and $M^{2}$ ), we have

$$
\langle u\rangle_{I}\langle v\rangle_{I} \geq\langle\sqrt{u v}\rangle_{I}^{2} \geq m^{2}, \quad I \subset[0,1] .
$$

To check the upper estimate, we fix $n$ and consider the same three types of intervals $\mathcal{D}_{1}, \mathcal{D}_{2}$, and $\mathcal{D}_{3}$ introduced in the proof of the preceding lemma. For $I \in \mathcal{D}_{1}$, the question reduces to the same question for smaller $n$ and, therefore, the estimate is true 
by induction, because $\left\langle u_{0}\right\rangle_{I}\left\langle v_{0}\right\rangle_{I}=M^{2}$. For $I \in \mathcal{D}_{2}$, we use the averages (7.8) calculated above:

$$
\begin{aligned}
\langle u\rangle_{I}\langle v\rangle_{I} & =\left(\frac{M}{a}-2^{k-N-1} \frac{M-m}{a}\right)\left(a M-2^{k-N-1} a(M-m)\right) \\
& \leq \frac{M}{a} \cdot a M=M^{2} .
\end{aligned}
$$

For $I=J=[0,1]$, we have $\langle u\rangle_{I}\langle v\rangle_{I}=x_{1} x_{2}=M^{2}$, and on all other intervals $I \in \mathcal{D}_{3}$ the functions $u, v$ are constants and $u v=m^{2}<M^{2}$.

The value of the sum in the definition of the Bellman function corresponding to the pair $u, v$ was calculated in Lemma 6 , hence for all values of $n$ and $N$ we have

$$
\begin{aligned}
\mathbf{B}(x ; m, M) & \geq b_{n}(N, m, M) \\
& =(M-m)\left(3 M-m-(M-m) 2^{-N+1}\right)\left(1-\left(1-2^{-N}\right)^{n}\right) .
\end{aligned}
$$

Passing first to the limit as $n \rightarrow \infty$ and then as $N \rightarrow \infty$, we get

$$
\mathbf{B}(x ; m, M) \geq(M-m)(3 M-m)=\mathcal{B}(x ; m, M) \quad \text { if } x_{1} x_{2}=M^{2} .
$$

So, we get an extremal sequence of pairs $u, v$ for an arbitrary point $x$ on the upper boundary of the domain $\Omega$. It remains to refer to Remark 10, where it was explained how to get an extremal pair for a point inside $\Omega$ if such a pair is known for the points of the upper boundary.

The main difficulty in obtaining a similar statement for the nondyadic Bellman function consists in verifying the upper estimate (2.1) for the pair of test functions, because we need to check this estimate for all subintervals of $[0,1]$. For this more difficult estimate we use the following lemma.

Lemma 8. If $a<2$, then

$$
\begin{array}{ll}
\left\langle u_{n}\right\rangle_{[0, t]} \leq \frac{a^{2}+1}{2 a} C, \quad\left\langle v_{n}\right\rangle_{[0, t]} \leq \frac{a C}{2-a} \\
\left\langle u_{n}\right\rangle_{[t, 1]} \leq \frac{a C}{2-a}, & \left\langle v_{n}\right\rangle_{[t, 1]} \leq \frac{a^{2}+1}{2 a} C .
\end{array}
$$

Proof. Since $v_{n}(t)=u_{n}(1-t)$, it suffices to check only one pair of estimates, for example, (7.10). This will be done by induction. For $n=0$, the estimates are true because $u_{0}(t)=v_{0}(t)=C$ and $a>1$. We assume that (7.10) is true for some $n$ and check it for the next value of the index, by using relations (7.2) $-(7.3)$.

For $t \in\left[0,2^{-N-1}\right]$ we have

$$
\begin{aligned}
& \left\langle u_{n+1}\right\rangle_{[0, t]}=\frac{m}{a}<\frac{a^{2}+1}{2 a} m<\frac{a^{2}+1}{2 a} C, \\
& \left\langle v_{n+1}\right\rangle_{[0, t]}=a m<\frac{a m}{2-a}<\frac{a C}{2-a} .
\end{aligned}
$$


If $t \in\left[2^{-k-1}, 2^{-k}\right], 1 \leq k \leq N$, then

$$
\begin{aligned}
\left\langle u_{n+1}\right\rangle_{[0, t]} & =\frac{1}{t}\left[2^{-N-1} \frac{m}{a}+\left(2^{-k-1}-2^{-N-1}\right) \frac{C}{a}+\int_{2^{-k-1}}^{t} u_{n+1}(s) d s\right] \\
& \leq \frac{1}{t}\left[2^{-k-1} \frac{C}{a}+\frac{1}{a} \int_{2^{-k-1}}^{t} u_{n}\left(2^{k+1} s-1\right) d s\right] \\
& \leq \frac{2^{-k-1}}{t}\left[\frac{C}{a}+\frac{1}{a} \int_{0}^{2^{k+1} t-1} u_{n}(s) d s\right] \\
& \leq \frac{2^{-k-1}}{t}\left[\frac{C}{a}+\left(2^{k+1} t-1\right) \frac{a^{2}+1}{2 a^{2}} C\right] \\
& \leq \frac{1}{2}\left[\frac{C}{a}+\frac{a^{2}+1}{2 a^{2}} C\right] \leq \frac{a^{2}+1}{2 a} C ; \\
\left\langle v_{n+1}\right\rangle_{[0, t]} & =\frac{1}{t}\left[2^{-N-1} a m+\left(2^{-k-1}-2^{-N-1}\right) a C+\int_{2^{-k-1}}^{t} v_{n+1}(s) d s\right] \\
& \leq \frac{1}{t}\left[2^{-k-1} a C+a \int_{2^{-k-1}}^{t} v_{n}\left(2^{k+1} s-1\right) d s\right] \\
& \leq \frac{2^{-k-1}}{t}\left[a C+a \int_{0}^{2^{k+1}} t-1\right. \\
& \leq \frac{\left.2^{-k-1}(s) d s\right]}{t}\left[a C+\left(2^{k+1} t-1\right) \frac{a^{2}}{2-a} C\right] \\
& \leq \frac{1}{2}\left[a C+\frac{a^{2}}{2-a} C\right]=\frac{a C}{2-a} .
\end{aligned}
$$

If $t \in\left[1-2^{-k}, 1-2^{-k-1}\right], 1 \leq k \leq N$, then

$$
\begin{aligned}
\left\langle u_{n+1}\right\rangle_{[0, t]}= & \frac{1}{t}\left[2^{-N-1} \frac{m}{a}+\left(\frac{1}{2}-2^{-N-1}\right) \frac{C}{a}\right. \\
& \left.+\left(\frac{1}{2}-2^{-k}\right) a C+\int_{1-2^{-k}}^{t} u_{n+1}(s) d s\right] \\
\leq & \frac{1}{t}\left[\frac{C}{2 a}+\left(\frac{1}{2}-2^{-k}\right) a C+\left(t-1+2^{-k}\right) \frac{a^{2}+1}{2} C\right] \\
\leq & \frac{1}{1-2^{-k-1}}\left[\frac{C}{2 a}+\left(\frac{1}{2}-2^{-k}\right) a C+2^{-k-1} \frac{a^{2}+1}{2} C\right] \leq \frac{a^{2}+1}{2 a} C ; \\
\left\langle v_{n+1}\right\rangle_{[0, t]}= & \frac{1}{t}\left[2^{-N-1} a m+\left(\frac{1}{2}-2^{-N-1}\right) a C\right. \\
& \left.+\left(\frac{1}{2}-2^{-k}\right) \frac{C}{a}+\int_{1-2^{-k}}^{t} v_{n+1}(s) d s\right] \\
\leq & \frac{1}{t}\left[\frac{1}{2} a C+\left(\frac{1}{2}-2^{-k}\right) \frac{C}{a}+\left(t-1+2^{-k}\right) \frac{C}{2-a}\right] \\
\leq & \frac{1}{1-2^{-k-1}}\left[\frac{1}{2} a C+\left(\frac{1}{2}-2^{-k}\right) \frac{C}{a}+2^{-k-1} \frac{C}{2-a}\right] \leq \frac{a C}{2-a} .
\end{aligned}
$$

Finally, if $t \in\left[1-2^{-N-1}, 1\right]$, where $u_{n+1}(t)=a m$ and $v_{n+1}(t)=\frac{m}{a}$ are constant functions, then

$$
\begin{aligned}
& C=\left\langle u_{n+1}\right\rangle_{[0,1]}=t\left\langle u_{n+1}\right\rangle_{[0, t]}+(1-t) \text { const } \\
& C=\left\langle v_{n+1}\right\rangle_{[0,1]}=t\left\langle v_{n+1}\right\rangle_{[0, t]}+(1-t) \text { const }
\end{aligned}
$$


i.e.,

$$
\left\langle u_{n+1}\right\rangle_{[0, t]}=\frac{C-(1-t) \text { const }}{t} \text { and }\left\langle v_{n+1}\right\rangle_{[0, t]}=\frac{C-(1-t) \text { const }}{t}
$$

are monotone functions that attain their maximal values at an end of the interval, where the required estimates are true.

Lemma 9. If $a<\sqrt{2}$, then

$$
\left\langle u_{n}\right\rangle_{I}\left\langle v_{n}\right\rangle_{I} \leq a^{6} C^{2}, \quad I \subset[0,1] .
$$

Proof. To make the calculations simpler, we replace estimates (7.10)-(7.11) by the following:

$$
\begin{aligned}
& \left\langle u_{n}\right\rangle_{[0, t]} \leq a^{3} C, \quad\left\langle u_{n}\right\rangle_{[t, 1]} \leq a^{3} C, \\
& \left\langle v_{n}\right\rangle_{[0, t]} \leq a^{3} C, \quad\left\langle v_{n}\right\rangle_{[t, 1]} \leq a^{3} C .
\end{aligned}
$$

The inequality

is true for all $a$, but the second inequality

$$
\frac{a^{2}+1}{2 a}<a^{3}
$$

$$
\frac{a}{2-a}<a^{3}
$$

is true for $a$ sufficiently close to 1 , more precisely, for $a^{2}<a+1$. Indeed, multiplying this inequality by $a-1$, we get

$$
a^{3}-a^{2}<a^{2}-1, \quad 1<a^{2}(2-a), \quad \frac{a}{2-a}<a^{3} .
$$

Now we start proving the claim of the lemma by induction. For $n=0$, it is obvious:

$$
\left\langle u_{0}\right\rangle_{I}\left\langle v_{0}\right\rangle_{I}=C^{2} \leq a^{6} C^{2} .
$$

Suppose it is true for some $n$; we prove the claim for the next value of the index. We introduce the following notation: $I_{k}^{-}=\left[2^{-k-1}, 2^{-k}\right], I_{k}^{+}=\left[1-2^{-k}, 1-2^{-k-1}\right]$ for $1 \leq k \leq N$, and $I_{N+1}^{-}=\left[0,2^{-N-1}\right], I_{N+1}^{+}=\left[1-2^{-N-1}, 1\right]$. First, we note that if $I \subset I_{k}^{ \pm}$, then the required inequality is true by the inductive hypothesis for $k \leq N$ and is obvious for $k=N+1$, where our functions coincide with the constants $a m$ and $\frac{m}{a}$.

So, we need to prove the estimate only for $I$ that have nonempty intersection with more than one interval $I_{k}^{ \pm}$. By formulas (17.6), the averages $\left\langle u_{n+1}\right\rangle_{I_{k}^{ \pm}}$and $\left\langle v_{n+1}\right\rangle_{I_{k}^{ \pm}}$are either $a C$ or $\frac{C}{a}$. By estimate (7.12), the averages over the end parts of $I$, which are noncomplete intervals $I_{k}^{ \pm}$, do not exceed $a^{3} C$; hence for the whole interval $I$ we have $\left\langle u_{n+1}\right\rangle_{I} \leq a^{3} C,\left\langle v_{n+1}\right\rangle_{I} \leq a^{3} C$, and the lemma is proved.

Lemma 10. $\mathbf{B}(x ; m, M) \geq \mathcal{B}(x ; m, M)$ for all $x \in \Omega_{m, M}$ such that $x_{1} x_{2}<M^{2}$.

Proof. We choose an arbitrary $C, \sqrt{x_{1} x_{2}}<C<M$, and take $N$ sufficiently large to have $a$ sufficiently close to 1 , so that $a^{3} C \leq M$. Then we define two test functions $u$ and $v$ on the interval $J=[-\rho, 1]$, where

$$
\rho=\frac{C-\sqrt{x_{1} x_{2}}}{\sqrt{x_{1} x_{2}}-m}
$$

as follows:

$$
u(t)=\left\{\begin{array}{ll}
\sqrt{\frac{x_{1}}{x_{2}}} m, & t<0, \\
\sqrt{\frac{x_{1}}{x_{2}}} u_{n}(t), & t \geq 0,
\end{array} \quad \text { and } \quad v(t)= \begin{cases}\sqrt{\frac{x_{2}}{x_{1}}} m, & t<0 \\
\sqrt{\frac{x_{2}}{x_{1}}} v_{n}(t), & t \geq 0\end{cases}\right.
$$


By direct calculation we check the averages of $u$ and $v$ :

$$
\langle u\rangle_{J}=\frac{\rho}{\rho+1} \cdot \sqrt{\frac{x_{1}}{x_{2}}} m+\frac{1}{\rho+1} \cdot \sqrt{\frac{x_{1}}{x_{2}}} C=x_{1}
$$

and in a similar way, $\langle v\rangle_{J}=x_{2}$. In all our examples the lower estimate $\langle u\rangle_{I}\langle v\rangle_{I} \geq m^{2}$ is trivial, because we always have $u(t) v(t) \geq m^{2}$. Lemma 9 provides us with the upper estimate $\langle u\rangle_{I}\langle v\rangle_{I} \leq M^{2}$, i.e., this pair satisfies condition (2.1).

Now we choose the following partition of the interval $J: J^{-}=[-\rho, 0], J^{+}=[0,1]$, and then the dyadic partition for $J^{ \pm}$, i.e,

$$
\alpha^{-}=\frac{\rho}{1+\rho}=\frac{C-\sqrt{x_{1} x_{2}}}{C-m}, \quad \alpha^{+}=\frac{1}{1+\rho}=\frac{\sqrt{x_{1} x_{2}}-m}{C-m},
$$

in the first generation and $\alpha^{-}=\alpha^{+}=\frac{1}{2}$ in all other generations. Calculating the sum in the definition of $\mathbf{B}$, we recall that the sum over the interval $[0,1]$ was calculated in Lemma 6.

$$
\begin{aligned}
\mathbf{B}(x ; m, & M) \\
\geq & |J|^{-1} \sum_{I \in \mathcal{I}}\left|\left(u, h_{I}\right)\right|\left|\left(v, h_{I}\right)\right| \\
= & \frac{\rho}{1+\rho} \cdot \frac{1}{1+\rho}\left|\langle u\rangle_{[-\rho, 0]}-\langle u\rangle_{[0,1]}\right|\left|\langle v\rangle_{[-\rho, 0]}-\langle v\rangle_{[0,1]}\right|+\frac{1}{1+\rho} b_{n}(N, m, C) \\
= & \frac{C-\sqrt{x_{1} x_{2}}}{C-m} \cdot \frac{\sqrt{x_{1} x_{2}}-m}{C-m}(C-m)^{2}+\frac{\sqrt{x_{1} x_{2}}-m}{C-m} b_{n}(N, C, m) \\
= & \left(C-\sqrt{x_{1} x_{2}}\right)\left(\sqrt{x_{1} x_{2}}-m\right) \\
& +\left(\sqrt{x_{1} x_{2}}-m\right)\left(3 C-m-(C-m) 2^{-N+1}\right)\left(1-\left(1-2^{-N}\right)^{n}\right) .
\end{aligned}
$$

Passing to the limit as $n \rightarrow \infty$ and then as $N \rightarrow \infty$, we get

$$
\begin{aligned}
\mathbf{B}(x ; m, M) & \geq\left(C-\sqrt{x_{1} x_{2}}\right)\left(\sqrt{x_{1} x_{2}}-m\right)+\left(\sqrt{x_{1} x_{2}}-m\right)(3 C-m) \\
& =\left(\sqrt{x_{1} x_{2}}-m\right)\left(4 C-\sqrt{x_{1} x_{2}}-m\right)=\mathcal{B}(x ; m, C) .
\end{aligned}
$$

Since $C$ can be chosen arbitrarily close to $M$, we can pass to the limit as $C \rightarrow M$ and obtain

$$
\mathbf{B}(x ; m, M) \geq \mathcal{B}(x ; m, M) .
$$

This argument completes the proof of Theorem 3 for all interior points of the domain $\Omega$.

\section{§8. CONCLUding REMARKS}

First, we briefly discuss an obstruction for a similar example of an extremal sequence for the boundary points $x$ with $x_{1} x_{2}=M^{2}$.

Recall that, essentially, our inductive procedure of constructing an extremal sequence consists in the following: every interval where at the preceding step the functions were constant $\left(u_{n}=x_{1}\right.$ and $\left.v_{n}=x_{2}\right)$ is split into four pieces, after which the pair $u_{n}, v_{n}$ is replaced by a pair $u_{n+1}, v_{n+1}$ of step functions, where $u_{n+1}$ has four steps

$$
\left\{\frac{m}{a} \sqrt{\frac{x_{1}}{x_{2}}}, \frac{1}{a} x_{1}, a x_{1}, m a \sqrt{\frac{x_{1}}{x_{2}}}\right\}
$$

and $v_{n+1}$ has four corresponding steps

$$
\left\{m a \sqrt{\frac{x_{2}}{x_{1}}}, a x_{2}, \frac{1}{a} x_{2}, \frac{m}{a} \sqrt{\frac{x_{2}}{x_{1}}}\right\} .
$$

However, this procedure never gives a pair satisfying (2.1) for all $I$, because the product of averages over the interval $I$ that is the union of two central parts among the four parts of our partition is always greater than $M^{2}$. It seems that we can improve the situation 
if we put points on the lower boundary in the middle of the interval to be split, i.e., take the steps for $u_{n+1}$ in the following order: $\left\{\frac{1}{a} x_{1}, \frac{m}{a} \sqrt{x_{1} / x_{2}}, m a \sqrt{x_{1} / x_{2}}, a x_{1}\right\}$, and for $v_{n+1}$, respectively, $\left\{a x_{2}, m a \sqrt{x_{2} / x_{1}}, \frac{m}{a} \sqrt{x_{2} / x_{1}}, \frac{1}{a} x_{2}\right\}$. Nevertheless, such a change does not help much. The first few steps yield a sequence satisfying condition (2.1), but not up to infinity.

Note that if we have a couple of step functions $u$ and $v$ on $J$ that are equal to constants $u^{ \pm}$and $v^{ \pm}$on $J^{ \pm}$, then the point $\left\{\langle u\rangle_{I},\langle v\rangle_{I}\right\}$ runs over the entire interval with endpoints $\left(u^{ \pm}, v^{ \pm}\right)$. Therefore, if condition (2.1) is fulfilled for all intervals $I \subset J$, then the points corresponding to adjacent intervals cannot be arbitrarily far from each other. But if we fix any interval corresponding to a point on the lower boundary, and try to check how its neighbor will change in our iterative process, then we see that it is farther and farther from the starting point. This means that for a fixed $N$ (or a fixed $a$ ) we cannot make arbitrarily many steps, i.e., we cannot make $n$ arbitrarily large to kill the term $\left(1-2^{-N}\right)^{n}$ in the expression for $b_{n}$. Moreover, a careful calculation shows that if for a given $N$ we take the maximal possible $n=n(N)$ such that the condition (2.1) is still fulfilled, then the expression $\left(1-2^{-N}\right)^{n(N)}$ does not tend to zero as $N$ tends to infinity.

For this reason, we shall not be too surprised if it happens that in the continuous case the Bellman function $\mathbf{B}$ is not continuous on the upper boundary and is smaller than $3 M^{2}-4 M m+m^{2}$.

The second remark is related to the proof of the main inequality (4.3) in Lemma 4 . We proved it using a specific expression for the candidate for the role of the Bellman function, but methodologically it would be interesting to deduce this global inequality from its infinitesimal form (4.4). This could be done by a direct integration only for the triples $x^{-}, x, x^{+}$such that the entire straight line segment with the endpoints $x^{ \pm}$is in $\Omega$.

\section{REFERENCES}

[B] D. L. Burkholder, Explorations in martingale theory and its applications, École d'Été de Probabilités de Saint-Flour XIX-1989, Lecture Notes in Math., vol. 1464, Springer, Berlin, 1991, pp. 1-66. MR.1108183 (92m:60037)

[CS] M. Cotlar and C. Sadosky, On the Helson-Szegö theorem and a related class of modified Toeplitz kernels, Harmonic Analysis in Euclidean Spaces (G. Weiss and S. Wainger, eds.) (Proc. Sympos. Pure Math., Williams Coll., Williamstown, MA, 1978), Part 1, Proc. Sympos. Pure Math., vol. 35, Amer. Math. Soc., Providence, RI, 1979, pp. 383-407. MR545279 (81j:42022)

[N] F. Nazarov, A counterexample to a problem of Sarason on boundedness of the product of two Toeplitz operators, Preprint, 1996, pp. 1-5.

[NTV1] F. Nazarov, S. Treil, and A. Volberg, The Bellman functions and two-weight inequalities for Haar multipliers, J. Amer. Math. Soc. 12 (1999), 909-928. MR.1685781 (2000k:42009)

[NTV2] — The T1 theorem for individual Haar multiplier, Preprint, 2004.

[NV] F. Nazarov and A. Volberg, The Bellman function, the two-weight Hilbert transform, and embeddings of the model spaces $K_{\theta}$. Dedicated to the memory of Thomas H. Wolff, J. Anal. Math. 87 (2002), 385-414. MR1945290 (2003j:30081)

[S1] E. T. Sawyer, A characterization of a two-weight norm inequality for maximal operators, Studia Math. 75 (1982), 1-11. MR676801(84i:42032)

[S2] - A characterization of two weight norm inequalities for fractional and Poisson integrals, Trans. Amer. Math. Soc. 308 (1988), 533-545. MR930072 (89d:26009)

St. Petersburg Branch, Steklov Mathematical Institute, Russian Academy of Sciences, Fontanka 27, St. Petersburg 191023, Russia

E-mail address: vasyunin@pdmi.ras.ru

Department of Mathematics, Michigan State University, East Lansing, Michigan 48824

E-mail address: volberg@yandex.ru

Received 30/NOV/2005

Translated by THE AUTHORS 